Pierre Brunet

\title{
Introduction : la hiérarchie des normes, fétiche ou nécessité ?
}

\begin{abstract}
Warning
The contents of this site is subject to the French law on intellectual property and is the exclusive property of the publisher.

The works on this site can be accessed and reproduced on paper or digital media, provided that they are strictly used for personal, scientific or educational purposes excluding any commercial exploitation. Reproduction must necessarily mention the editor, the journal name, the author and the document reference.

Any other reproduction is strictly forbidden without permission of the publisher, except in cases provided by legislation in force in France.
\end{abstract}

\section{revues.org}

Revues.org is a platform for journals in the humanities and social sciences run by the CLEO, Centre for open electronic publishing (CNRS, EHESS, UP, UAPV).

Electronic reference

Pierre Brunet, «Introduction : la hiérarchie des normes, fétiche ou nécessité ? », Revus [Online], 21 | 2013, Online since 25 February 2014, connection on 03 March 2014. URL : http://revus.revues.org/2768

Publisher: Klub Revus - Center za raziskovanje evropske ustavnosti in demokracije http://revus.revues.org

http://www.revues.org

Document available online on: http://revus.revues.org/2768

This document is a facsimile of the print edition.

All rights reserved 


\section{Introduction : la hiérarchie des normes, fétiche ou nécessité?}

L'expression " hiérarchie des normes » fait sans aucun doute partie des fétiches des juristes. Introduite par la science du droit, l'expression est, depuis, passée dans le langage ordinaire des juristes au point que certains en sont venus à croire qu'elle désigne une réalité objective, une sorte de nature du droit voire d'essence de la juridicité. Or, comme les textes qu'on va lire permettent de le montrer, l'expression « hiérarchie des normes » est bien plus ambiguë qu'on ne le croit. Elle est susceptible dêtre utilisée pour désigner tantôt une théorie, tantôt un argument juridique et parfois même les deux à la fois.

La théorie de la hiérarchie des normes est bien connue. Elle tient au fait que le droit organise et règle sa propre création de sorte que l'ordre juridique ne consiste pas en un ensemble de normes même coordonnées entre elles qui se situeraient toutes sur le même plan mais en un ensemble de normes entretenant entre elles un rapport de création/application : une norme est créée selon une procédure elle-même déterminée par une autre norme de sorte que la première norme sert de fondement de validité à une seconde norme. Si la validité de la seconde norme est fondée sur la validité de la première, on peut conclure que ces deux normes entretiennent entre elles un rapport hiérarchique. En d'autres termes, les normes qui règlent la création d’autres normes sont dites supérieures à celles-ci, lesquelles, à leur tour sont dites inférieures à celles-là.

Si elle est désormais indissolublement liée au nom de Kelsen, elle ne saurait lui être seulement imputable car elle doit énormément au travail pionnier de Merkl. Nul ne peut prétendre savoir si Kelsen serait parvenu à cette formalisation de la " double face » du droit, simultanément création et application, sans l'aide de Merkl. Il ne fait en revanche guère de doute que Kelsen a pu très vite mesurer la nécessité de sortir d'une représentation statique du droit pour rendre compte de sa dimension dynamique. Il reste que, comme Stanley Paulson le montre fort bien, il ne faudrait pas croire que le concept dynamique de norme ou d'ordre juridique s'oppose voire contredit le concept statique : les deux se complètent dans un «concept unique " qui reflète à la fois la contrainte propre au droit et l'acte de création que toute contrainte suppose et implique simultanément.

Ainsi, la contribution de Stanley Paulson nous permet-elle, indirectement et c'est une autre de ses vertus, de saisir l'ambivalence du terme "statique » que Kelsen utilise à la fois pour désigner les éléments composant tout ordre juridique (le sujet de droit, la sanction, la représentation...), et un certain mode de production du droit par déduction typique de la conception jusnaturaliste, dans lequel la volonté de celui qui pose les normes n’entre jamais en ligne de compte. 
A cet égard, il est incontestable que l'opposition entre le statique et le dynamique pour ce qui concerne le mode de production des normes est certainement un des apports fondamentaux de la théorie de Kelsen et d'une richesse heuristique considérable. C'est bien cette théorie qui nous permet de comprendre le rôle considérable de la volonté en droit sans pour autant introduire dans la description de son objet des concepts empruntés au droit lui-même - tel celui d'obligation - ou à la sociologie - comme pour le concept d'autorité.

Mais de quelle « hiérarchie » parle-t-on et quel concept de hiérarchie utilise-t -on quand on parle de hiérarchie des normes ? Kelsen et Merkl envisageaient une hiérarchie formelle, celle qui existe entre les normes réglant la création du droit et le droit créé conformément à celles-ci. Il n’empêche que cette hiérarchie formelle a elle-même soulevé nombre d’objections dont celle, logique, selon laquelle Kelsen aurait confondu la validité de l'inférence logique d'une norme à une autre avec l'inférence de la validité juridique. Or, d'une part, la validité juridique d'une norme ne peut en aucun cas être « logiquement » inférée de la validité juridique de la norme supérieure et d'autre part, l'inférence logique d'une norme à partir d'une autre ne nous permet pas de conclure nécessairement à l'inférence de sa validité juridique. En d'autres termes, la validité de l'inférence - c'est-à-dire la conformité d'une inférence à des règles logiques - ne se confond pas avec l'inférence de la validité - c'est-à-dire l'inférence de la validité juridique conformément aux règles du système juridique.

Mais, comme le montre Riccardo Guastini, une analyse logique permet en outre d'identifier trois autres concepts de « hiérarchie » qui peuvent être utilisés par les juristes : une hiérarchie matérielle - lorsqu'une norme positive interdit à deux autres normes de se contredire ; une hiérarchie logique - lorsqu'une norme porte sur l'autre d'un point de vue méta-linguistique ; et enfin une hiérarchie axiologique qui dépend d'un jugement de valeur des interprètes lesquels attribuent à une norme une valeur supérieure à une autre.

Comme le montre la contribution de Rafael Encinas de Munagorri, la hiérarchie des normes consiste à rendre compte d'une façon spécifique et singulière de produire du droit à laquelle il est bien difficile d'échapper. Elle a certes été parfois comprise, à tort, comme une doctrine idéologique visant à établir la supériorité axiologique d'une norme sur une autre. Ainsi, parce que les méthodes traditionnelles du droit international privé pour résoudre les conflits de lois et les conflits de juridictions ont été forgées en dehors de la hiérarchie des normes, certains spécialistes du droit international privé ont pu estimer que l'idée même de hiérarchie des normes était inappropriée au sein de leur discipline. Mais cétait se méprendre doublement : à la fois sur le concept de hiérarchie tel que l'utilisait Kelsen et sur celui qu'utilisaient ces juristes de droit international privé. 
Ce type de méprise n'est pas rare dès lors que les concepts dont on fait usage ne sont pas eux-mêmes soumis à une critique scrupuleuse et que l'on ne tient pas la bonne distance entre le métalangage du juriste et le langage du droit. La confrontation des modèles de contrôle de constitutionnalité en fournit une bonne illustration. Comme le montre Guillaume Tusseau, l'opposition entre un modèle européen et un modèle américain de justice constitutionnelle n'est, à l'analyse, guère défendable et mérite dêtre remplacée par un nouveau mode d'analyse qui soit justement conscient de la relativité des points des vue et du danger que court toute universalisation.

On ne saurait non plus réduire la hiérarchie des normes à ce rapport d'autorité que l'on impute bien souvent au premier positivisme formaliste. On pense ici à la lecture de Austin par Hart et sa distinction capitale entre normes primaires et secondaires sur laquelle revient Massimo La Torre. On sait en effet que Hart rejette la représentation "impérativiste » du droit selon laquelle le droit serait constitué de commandements imposant l'obéissance à leurs destinataires car cette thèse ne parvient pas à rendre compte en termes juridiques du pouvoir dont dispose celui qui pose les commandements. D'où l'importance de la distinction entre des normes dites primaires qui imposent des comportements et celles dites secondaires qui attribuent des pouvoirs et organisent le fonctionnement des normes primaires. Cette distinction permet déviter l'aporie de la représentation impérativiste. Mais cette distinction établit aussi une double hiérarchie, à la fois formelle et logique car les normes secondaires servent de fondement de validité en même temps qu'elles se rapportent aux normes primaires.

Dans ces conditions, on semble parfaitement fondé à rapprocher la représentation normativiste de Kelsen-Merkl et celle de Hart. Et le point de vue interne qui est si souvent invoqué à propos de Hart et dont il est l'un des ardents défenseurs est tout autant celui adopté par Kelsen lequel ne parle certes pas de "point de vue interne » mais d'un " point de vue normatif» dont Bobbio a su montrer qu'il était simultanément descriptif et interprétatif car relevant d'une explication sans causalité. C'est d'ailleurs ce qui explique que Kelsen ait eu besoin de la fiction de la norme fondamentale laquelle, comme il le reconnaissait très bien, est un présupposé nécessaire à quiconque entend conférer une signification objective de norme à un acte qui n'a qu'une signification subjective de norme. De sorte que cette fiction est certes une fiction de la science du droit mais une fiction à laquelle est également contrainte toute autorité qui entend poser une norme préalablement à cet acte de volonté. Dans ces conditions, et dans ce contexte, il n'y a pas grande différence entre présupposer et poser une norme (comme le reconnaissait Robert Walter).

On peut toutefois rendre compte autrement de cette nécessité, en adoptant un point de vue externe qui caractérise si bien les réalistes et les opposaient 
à Hart. Selon une analyse réaliste et empiriste, la théorie de la hiérarchie des normes peut être vue comme une théorie politique, explique Eric Millard, qui construit son objet comme contrainte dans l'argumentation juridique et qui a donc une dimension normative essentielle. Si l'on prolonge l'analyse, on peut montrer, comme le fait de son côté Raphaël Paour, que la théorie de la hiérarchie des normes fournit une ressource non négligeable pour contraindre les organes de concrétisation à prendre en compte leurs préférences à l'aide d'énoncés juridiques généraux et abstraits et ce d'autant plus qu'il existe une corrélation entre le pouvoir d'un organe et le rang hiérarchique des normes qu'il produit. De même, c'est encore ce point de vue réaliste qui permet à Luca Parisoli de saisir, dans l'argumentation des juristes médiévaux, la manipulation des contradictions déontiques prima facie associée à la théorie de la légitimité de la hiérarchie normative. Cette dernière peut toutefois être entendue non seulement comme instrument politique mais aussi et essentiellement comme un instrument de rationalité au sein d'une science juridique orientée vers une théologie politique. De son côté, Francesco Di Donato montre également comment les Parlements d'ancien régime - c'est-à-dire les juristes de la robe - ont su utiliser l'argument d'une hiérarchie des normes pour remettre en cause l'ordre juridique monarchique et créer une place pour le Parlement dans la production du droit.

Chaque contribution souligne donc, soit directement ou soit indirectement, la grande difficulté méthodologique à laquelle tout juriste doit faire face lorsqu'il est confronté au concept de hiérarchie des normes : faire la part entre l'usage descriptif ou cognitif et l'usage argumentatif ou justificatif de l'idée que les normes juridiques s'inscrivent dans une hiérarchie et qu'elles sont précisément juridiques non parce quelles réaffirment une quelconque hiérarchie de valeurs mais parce que leurs auteurs parviennent à établir qu'ils n’en sont que les acteurs, au sens de Hobbes : leurs paroles ne sont pas l'expression de leur volonté mais celle d'une entité qui les dépassent. Et ainsi fonctionne un système juridique : par imputation successive d'une volonté à un autre que soi dont on affirme qu'il nous est supérieur et que nous lui devons obéissance.

On l'aura compris, l'ensemble des contributions aborde un très grand nombre de questions clefs de la théorie du droit. On pourrait regretter qu'en dépit de leur richesse, les analyses n'aient pas abordé de front des questions très actuelles telle que la remise en cause de la hiérarchie des normes sous l'effet de l'internationalisation ou, mieux, de la globalisation des ordres juridiques voire de leur intégration progressive. Le regret ne saurait durer. D’abord parce que les contributions fournissent toutes une grille conceptuelle susceptible d'être réutilisée dans d'autres contextes que le leur. Ensuite, parce que, sur le fondement de bien des textes ici réunis, on se sent autorisé à nuancer ce que d'aucuns présentent comme un constat purement factuel et neutre. Le constat que l'on peut faire est certainement que nombre de juridictions aujourd'hui, en Europe notamment 
mais pas seulement, tiennent toutes un discours selon lequel la représentation du droit comme un ordre hiérarchisé de normes serait dépassée, qu’il conviendrait enfin de tenir compte de la grande proximité matérielle entre ordres juridiques pour comprendre quarrivé à certains niveaux de normes, la hiérarchie nest plus possible mais que se forme un réseau normatif, fait d'ailleurs de principes communs, et que l'agencement conduit à un ordre pluriel ou pluraliste. Ce discours fort rassurant ne manque toutefois pas d'éveiller quelque scepticisme car si les principes sont à ce point communs aux différents ordres, comment expliquer qu'ils aient besoin dêtre sans cesse redéfinis ? Et quand bien même on expliquerait que leur redéfinition est constitutive de ces principes, comment imaginer que cette entreprise puisse être descriptive et cognitive et nêtre que cela quand ceux qui en ont la charge doivent précisément décider du sens, de la valeur et de la portée de ces principes ? Si donc plusieurs décisions doivent intervenir, viendra avec elles la nécessité de les concilier. Et cette conciliation conduira inévitablement à devoir dissoudre préventivement ou résoudre curativement déventuelles contradictions et antinomies. Or, pour ce faire, il faudra désigner une autorité et l'investir d'une compétence qui fera d’elle une autorité supérieure aux autres. On aura ainsi fait revenir la hiérarchie que l'on croyait disparue. Autrement dit, il en va du "pluralisme » des ordres normatifs comme de la hiérarchie des normes dans certains cas : ce peut nêtre qu'un argument justifiant un certain type de relations entre normes lesquelles demeurent toutefois l'expression d'un rapport de force et d'une certaine distribution du pouvoir. Aussi paradoxal que cela paraisse, pour affirmer et conférer une certaine autorité à l'affirmation qu'il n'y a pas de hiérarchie entre certaines normes encore faut-il se situer au sommet d'une hiérarchie de pouvoirs eux-mêmes normatifs. C'est donc ainsi que se fabrique l'ordre juridique : à l'aide de raisonnements, de justifications et de constructions argumentatives.

\section{Pierre Brunet,}

Professeur de droit public à l'Université Paris Ouest-Nanterre La Défense, Directeur de l'UMR CNRS 7074, Centre de Théorie et Analyse du Droit, Membre de l'Institut Universitaire de France

NB : Ce volume doit son existence à la persévérance et l'endurance de Andrej Kristan, de Jérémy Mercier et de Matija Žgur sans lesquels il n'y aurait eu ni normes, ni hiérarchie... Je les en remercie chaleureusement, infiniment. 


\section{Bibliographie}

Norberto BOBBIO, 1970: "Sein" and "Sollen" in Legal Science. Archiv für Rechts und Sozialphilosophie (1970) 6. 7-29.

Rafael ENCINAS DE MUNAGORRI, 2013 : Droit international privé et hiérarchie des normes. Observations sur une rencontre. Revus (2013) 21.

Riccardo GUASTINI, 2013a : Lex superior. Pour une théorie des hiérarchies normatives. Revus (2013) 21.

_—, 2013b : Gerarchie normative. Revus (2013) 21.

Hans KELSEN, 1965: Professor Stone and the Pure Theory of Law. Stanford Law Review (1965) July. 1128-1157.

_- 2013: La fonction de la Constitution (1964). In Christophe Bouriau (Ed.), Les fictions du droit. Kelsen, lecteur de Vaihinger, Lyon: ENS Editions. 87-96.

Massimo LA TORRE, 2013a : Le modèle hiérarchique et le Concept de droit de Hart. Revus (2013) 21.

- - 2013b : The Hierarchical Model and H. L. A. Hart's Concept of Law. Revus (2013) 21.
Éric MILLARD, 2013 : La hiérarchie des normes. Une critique sur un fondement empiriste. Revus (2013) 21 .

Raphaël PAOUR, 2013 : Les contraintes juridiques de la hiérarchie des normes. Revus (2013) 21.

Luca PARISOLI, 2013 : Une approche volontariste du droit naturel et de la contradiction. Une façon de bâtir la notion de hiérarchie dans la pensée latine médiévale. Revus (2013) 21.

Stanley L. PAULSON, 2013a : Ce que nous apprend la Stufenbaulehre sur le concept de droit de Kelsen. Revus (2013) 21.

-_, 2013b : How Merkl's Stufenbaulehre Informs Kelsen's Concept of Law. Revus (2013) 21.

Guillaume TUSSEAU, 2013 : Sur le métalangage du comparatiste. De la prétention à la neutralité à l'engagement pragmatiste. Revus (2013) 21.

Robert WALTER, 2013 : Der Aufbau der Rechtsordnung. Eine rechtstheoretische Untersuchung auf Grundlage der Reinen Rechtslehre, Wien, Manz, 1974 (La struttura dell'ordinamento giuridico. Un'analisi teorico-giuridica in base all Dottrina pura del diritto. Trad italienne N. Bersier Ladavac. Genève: Thémis.) 